\title{
Evaporation Dynamics of Boron Dopants in Silicon
}

Jonathan Op de Beeck ${ }^{1}$, Christoph Freysoldt ${ }^{2}$, Ramya Cuduvally ${ }^{3}$, Jeroen Scheerder ${ }^{4}$, Richard J. H. Morris ${ }^{4}$, Paul van der Heide ${ }^{5}$, Wilfried Vandervorst ${ }^{6}$ and Claudia Fleischmann ${ }^{7}$

${ }^{1}$ Imec, Kapeldreef 75, 3001 Leuven, Belgium, Quantum solid-state physics group, KU Leuven, Celestijnenlaan 200D, 3001 Leuven, Belgium, Belgium, ${ }^{2}$ Max-Planck-Institut für Eisenforschung GmbH, Max-Planck-Straße 1, 40237 Düsseldorf, Germany, United States, ${ }^{3}$ Imec, Kapeldreef 75, 3001 Leuven, Belgium,Quantum solid-state physics group, KU Leuven, Celestijnenlaan 200D, 3001 Leuven, Belgium, United States, ${ }^{4}$ Imec, Kapeldreef 75, 3001 Leuven, Belgium, Leuven, Vlaams-Brabant, Belgium, ${ }^{5}$ Imec, Kapeldreef 75, 3001 Leuven, Belgium, United States, ${ }^{6}$ Imec, Kapeldreef 75, 3001 Leuven, Belgium, Quantum solid-state physics group, KU Leuven, Celestijnenlaan 200D, 3001 Leuven, Belgium, United States, ${ }^{7}$ Imec, Kapeldreef 75, 3001 Leuven, Belgium,Quantum solid-state physics group, KU Leuven, Celestijnenlaan 200D, 3001 Leuven, Belgium, Belgium

Albeit promising, atom probe tomography (APT) suffers from a loss in analytical performance in terms of resolution, accuracy and reliability when applied to semiconductors. A reoccurring theme is the dependence of the apparent chemical composition on the experimental analysis conditions (Cuduvally et al., 2020). These problems occur not only in complex heterogeneous systems, but also in diluted systems such as doped semiconductors (Martin \& Yatzor, 2019). In particular the quantification of B dopants within a silicon matrix has been studied intensively by the atom probe community as it is considered a key issue (Da Costa et al., 2012) due to its relevance for the semiconductor industry. The challenge is to propose physically motivated correction schemes which can be used even in more complex systems, e.g. nanodevices.

This work demonstrates that detailed multiple hit analyses in conjunction with field ion microscopy (FIM) experiments establish a powerful toolbox to reveal B-related cluster signals otherwise invisible to conventional APT analysis. Deciphering the origin of any incorrect quantification of dilute dopants is extremely challenging as it requires to sensitively tackle a comprehensive list of events leading up to detection. This includes atomic processes on the tip surface, evaporation and subsequent events during the ion flight, e.g. dissociation. In this study FIM provides an atomically resolved direct observation of the emitter surface. On the other hand, data analysis schemes such as ion-correlation histograms of filtered multiple hits reveal what arrives at the detector. The complementary nature of these approaches allows to a draw a more complete picture.

We shed light on possible sources of B quantification inaccuracies in APT analysis of B doped Si, with special focus on the preferential evaporation of B as a multiple event. In the latter multiple atoms are emitted and detected under a single stimulus (pulse), i.e. an increase in field or temperature. The preferential evaporation of B atoms in multiple events is quite surprising given the dopants are scarcely available which is expected to lead to a small probability for two B atoms to evaporate independently of each other in a single pulse. While typically attributed to co-evaporation (Da Costa et al., 2012), we propose clustering of $\mathrm{B}$ dopants and subsequent dissociation following field emission as a new and additional mechanism leading to an incorrect quantification and driving the anomalous multiplicity. Indeed, detailed analysis of the background reveals the presence of B cluster peaks never reported and lost in quantification (Figure 1a,b). Furthermore, the dissociation of such clusters which contributes to the high 
B multiplicity observed is demonstrated by the coulombic repulsion of the resulting daughter ions (Figure 1c).

A complete picture can only be drawn when accounting for the mechanisms behind the cluster formation, consisting of retention and migration phenomena. These can lead to erroneous quantification as they alter the B concentration across the tip surface artificially leading to over and under estimation of B (Figure 2). The atomic scale dynamics of the B atoms are investigated by FIM and APT and linked to their impact on B quantification. In perspective, the fundamental understanding of the evaporation physics for this model case is expected to improve quantification accuracy for systems in which dopant atoms possess a contrasting evaporation field with their matrix.
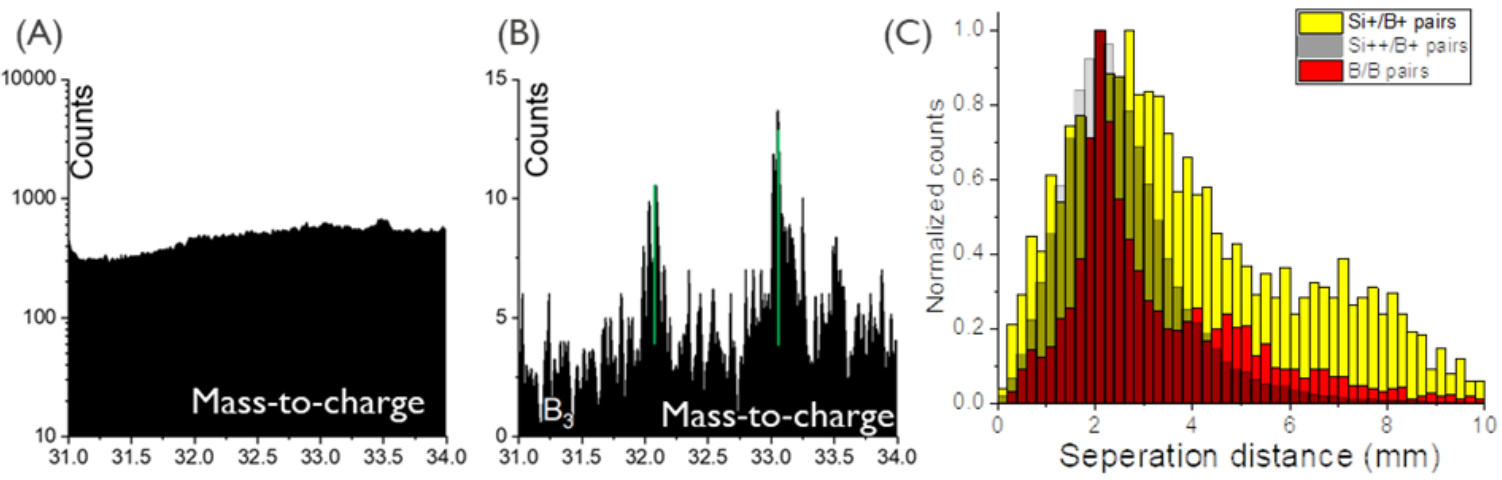

Figure 1. Direct evidence of B-related cluster formation during APT analysis in B doped Si. (A) Unfiltered mass spectrum indicating the background masking any peaks. (B) Filtered mass spectrum (multiplicity $\geq$ 4) revealing B3 peaks with matching isotopic abundance as indicated in green. (C) Evidence of coulombic repulsion for double hits resulting from cluster dissociation. A double peak in the histogram, representing the separation distance on the detector of ions in a double hit event, allows to separate co-evaporation (first peak) and molecular dissociation (second peak) contributions to multiple events.
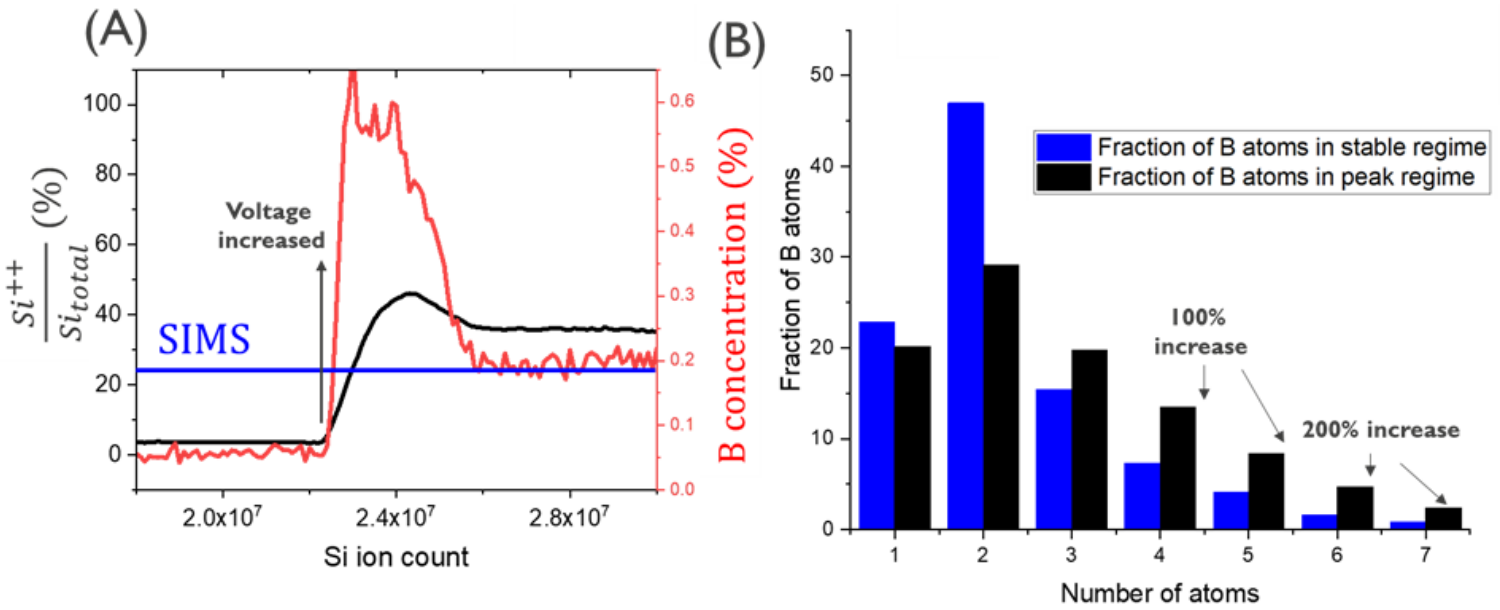

Figure 2. The retention of B atoms exemplified by suddenly increasing the applied voltage (hence field) to the emitter. (A) Overestimation of the B concentration. (B) Corresponding multiplicity histogram indicating large multiple events become particularly abundant (stable represents region of charge-stateratio of 0.36 ). 


\section{References}

Da Costa, G., Wang, H., Duguay, S., Bostel, A., Blavette, D. \& Deconihout, B. (2012). Advance in multi-hit detection and quantization in atom probe tomography. Review of Scientific Instruments 83, 123709.

Cuduvally, R., Morris, R. J. H., Ferrari, P., Bogdanowicz, J., Fleischmann, C., Melkonyan, D. \& VANDERVORST, W. (2020). Potential sources of compositional inaccuracy in the atom probe tomography of InxGa1-xAs. Ultramicroscopy 210, 112918.

MARTIN, A. J. \& YATZOR, B. (2019). Examining the Effect of Evaporation Field on Boron Measurements in SiGe: Insights into Improving the Relationship Between APT and SIMS Measurements of Boron. Microscopy and Microanalysis 25, 617-624. 